American Journal of Applied Sciences 7 (4): 493-499, 2010

ISSN 1546-9239

(c) 2010Science Publications

\title{
Individual Species Crown Mapping in Taman Rimba Ilmu, University Malaya Using Airborne Hyperspectral Imaging
}

\author{
Kamaruzaman Jusoff \\ Tropical Forest Airborne Observatory (TropAIR), Faculty of Forestry, \\ Universiti Putra Malaysia, 43400 Serdang, Selangor, Malaysia
}

\begin{abstract}
Problem statement: Accurate, current and cost-effective individual standing tree data are required by forest management communities for use in forest inventory over large areas. Currently, most of the forest mapping process is done directly on the ground using many technique such as the bearing-distance and also the other technique that use the computer software as a support, such as Tree Mapper $^{\mathrm{TM}}$. Instead of ground data collection and where there are difficulties in reaching the individual trees, hyper spectral remote sensing technology is the best option to map the tree positions. Approach: A novel approach to generating an individual tree crown mapping estimated for a lowland dipterocarp forest of Taman Rimba Ilmu, University Malaya, Kuala Lumpur using an airborne hyperspatial $\left(1 \mathrm{~m}^{2}\right.$ ground resolution) imager was presented. Results: A total of 297 individual tree crowns comprising of 83 Xylopia sp., 79 Ixonanthes sp., 56 Hevea sp., 15 Streblus elongates, 14 Pellacalyx sp., 12 Endospermum diadenum, 11 Macaranga gigantea, 10 Cratoxylum sp., 10 Cannarium sp. and 7 Ixonanthes icosandra were identified and delineated as individual polygons in a study area plot of 2 ha. Conclusion/Recommendations: It was found out that individual tree crowns in University Malaya can be detected and counted with reasonable field measured to image derived mapping accuracy of $98.65 \%$. This study implied that acceptable individual tree crown classification maps and algometric equations relating diameter at breast height (dbh) or crown area to biomass can be used to generate timber volume estimates with established crown-diameter-volume correlations.
\end{abstract}

Key words: Airborne remote sensing, rainforest, tree crown, inventory, conservation

\section{INTRODUCTION}

Tree mapping can be defined as the acquisition of the tree position on the earth either in term of latitude and longitude and also the Cartesian coordinate. In Malaysia, the Grid method is commonly used to map the trees especially in the permanent forest plot. The Forest Service, United States Department of Agriculture used several methods in tree mapping on the forest plot (Wilson, 2000). These methods included Sequential Target Mapping Method and also the Plot Origin Mapping Method. Currently, most of the forest mapping process is done directly on the ground using many technique such as the bearing-distance and also the other technique that use the computer software as a support, such as Tree Mapper $^{\mathrm{TM}}$. This technique converts the ground reading in terms of distance and bearing into tree position in terms of points, depending of the method of data collection that is being used.

Instead of ground data collection and where there are difficulties in reaching the individual trees, hyper spectral remote sensing technology is the best option to map the tree positions (Jusoff, 2006; 2007; Jusoff, 2009b; 2009c; 2009d; Jusoff and Mubeena, 2009; Mohd Hasmadi et al., 2010). Airborne hyper spectral imaging technique using a UPM-TropAIR's AISA sensor has proven to be able to meet the demand of such data in high quality, at fairly low cost and with an expeditious response (Jusoff and Yusoff, 2008). In western Switzerland, a Hyper spectral Map per (HyMap) showed the capacity of hyper spectral HyMap data with linear unmixing models as a very useful tool for automatically separating coniferous and deciduous stands and their mixture-grade, which is very important for forest management (Darvishsefat et al., 2002).

The purpose of this study is therefore to assess the capability of the UPM-TropAIR's AISA airborne hyper spectral imaging technique in mapping of the Taman Rimba Ilmu, University Malaya's forest at the individual species level.

\section{MATERIALS AND METHODS}

Taman Rimba Ilmu or "The Forest of Knowledge" which was established in 1974 is a tropical botanical 
garden occupying an area of 80 ha, set up in the University of Malaya campus in Kuala Lumpur, Malaysia (Fig. 1). It is modeled after a rain forest garden concept rather than a formal flower garden. Together with the living collections of over 1,600 species, the Taman Rimba Ilmu also houses the University Malaya herbarium and has its own Environmental Education Programmed since 2007. The herbarium (a reference library of preserved plant specimens) is Malaysia's largest university collection containing some 63,000 accessions. The Rimba Ilmu is a member of the Botanic Gardens Conservation International and the South East Asia Botanic Gardens Network. The study area of size two ha is a sub-plot in Taman Rimba Ilmu. It is a secondary forest, partly buffered by old rubber plantings and the living collections, mostly from Malaysia, are one of the most important biological conservatories in Malaysia.

UPM-TropAIR's AISA airborne hyper spectral system is a commercial hyper spectral sensor product (15 $\mathrm{kg}$ in total weight), a complete push-broom system, consisting of a hyper spectral sensor head, miniature Global Position System (GPS) sensor and data acquisition unit in a rugged PC with display unit and power supply, operated by Tropical Forest Airborne Observatory (TropAIR), Faculty of Forestry, UPM. It is a proven hyper spectral system that has been designed to collect accurate and reliable information of the earth surface (Jusoff, 2008a; 2008b; 2008c; Jusoff, 2009b; 2009c; 2009d; Jusoff and Ya acob, 2008). It is quick to install and remove from any aircraft and provide timely, accurate and reliable information (Jusoff, 2010).

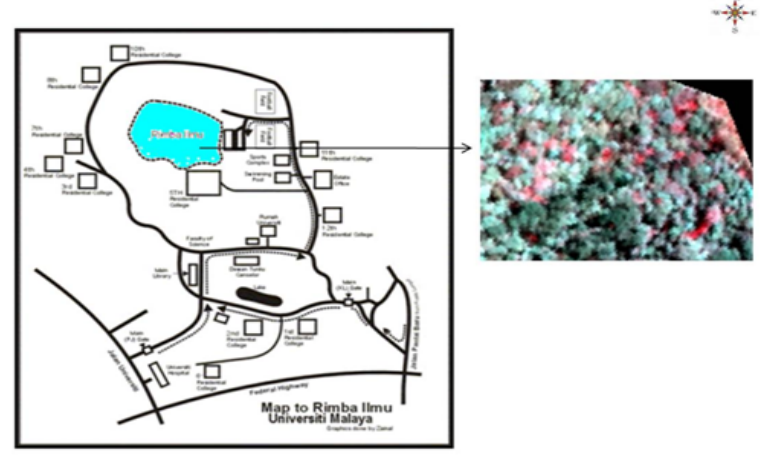

Not Drawn to Scale

Fig. 1: A map showing the study area in Taman Rimba Ilmu, University Malaya with the raw data sample acquired from the UPM-APSB's AISA sensor
The systems include an in-flight configuration setting, which allows alterations to be made easily for each exercise. Auxiliary components include a mount to connect the sensor head to the GPS or INS unit, regulated power supply and Cali geo post-processing software that produces calibrated geo-referenced images and image mosaics of the acquired data with an ENVI header. It is a ready-to-use system to produce radio metrically calibrated and geo-referenced hyper spectral data and measures up to 244 bands of contiguous visible and IR wavelengths at up to 100 images. UPM-TropAIR's AISA is capable of collecting data within a spectral range of $430-900 \mathrm{~nm}$. Although it is capable of collecting up to 286 spectral channels within this range, the data rate associated with the short integration times (sampling rates) required of the sensor in most operational or flight modes, limits the number of channels. The operational collection configuration used for this study is 20 spectral bands depending on the aircraft speed, altitude and mission goals.

Hyper spectral image of Taman Rimba Ilmu, University Malaya and its surrounding was acquired using UPM-TropAIR's AISA hyper spectral sensor. The sensor was flown at an altitude of $1000 \mathrm{~m}$ from the ground at a spatial resolution of $1.0 \mathrm{~m}$. The aircraft flying speed was 120 knots or $60 \mathrm{~m} \mathrm{sec}^{-1}$. Image preprocessing was done on-board automatically with the Cali geo software to increase the accuracy and the interpretability of the image prior to image classification. This process involves correcting images to reduce the magnitude of unwanted effects to improve the quality of the image data for subsequent processing in addition to correct for sensor and platform-specific radiometric and geometric distortions of data. Image enhancement using the contrast and optimum band combinations were later performed using ENVI 4.2 to edit the original image data to increase the amount of information for visually interpreted data to create a "new" image. In order to automatically extract the individual species pixels, the spectral signatures of the individual tree species must be analyzed and pixels were then assigned to categories based on similar spectral signatures. A supervised classification using Spectral Angle Map per (SAM) was finally applied to separate the end members for each tree crown species and later verified on the ground by the field survey team members from TropAIR using a handheld Global Positioning System (GPS). The supervised classification was done using the Spectral Angle Map per (SAM), which is a physically-based spectral classification that uses an n-dimensional angle to match pixels to reference spectra. The end-member of the tree crowns were derived from the archived spectral library, 
which were collected during the ground sampling using a handheld spectroradiometer. The radian used in this study was 0.10 , which is the most suitable radian to classify the species-species crowns of trees in the study area. A post classification using the clump classes was used to clump adjacent similar classified pixels together using morphological operators. The selected classes were then clumped together by first performing a dilate operation following by erode operation on the classified image using a kernel of the size specified in the parameters dialog. Sieve classes removed isolated classified pixels in classification image using blob grouping. The sieve classes at the neighboring 4 or 8 pixels were to determine that the pixel is grouped with pixels of the same class. The number of pixels in a class that were grouped less than the neighboring pixels value was removed away from the class. The mapping accuracy was calculated using following formula:

$$
\text { Persentage of accuracy }=\frac{\begin{array}{c}
\text { No. of trees det ected via } \\
\text { ground verification }
\end{array}}{\begin{array}{l}
\text { No. of trees mapped from } \\
\text { airborne hyperspectral image }
\end{array}} \times 100
$$

Finally, an output thematic map of individual tree crowns in Taman Rimba Ilmu, University Malaya was developed.

\section{RESULTS}

An optimal band combination of R12, G19 and B16 was found to be the best combination and was used for advance image analysis in the image classification procedure. Figure $2 \mathrm{a}$ and $2 \mathrm{~b}$, respectively shows the image of forest in Taman Rimba Ilmu before and after $80 \%$ image adds back (Sobel edge detection). Figure 3a shows that the distribution of 10 tree species in the study area after classification using SAM meanwhile Fig. $3 b$ shows the result after using clumping and sieving class for the 10 identified and mapped tree species in the study area. Based from a supervised classification, the 10 different classes of tree species successfully classified and mapped according to their matched spectral signatures are as follows:

$\begin{array}{ll}\text { Pella calyx sp. (Membuluh) } & \text { - White } \\ \text { Endospermum diadenum (Sesenduk) } & \text { - Green } \\ \text { Ixonanthes sp. (Inggir burung) } & \text { - Red } \\ \text { Cratoxylum sp. (Geronggan) } & \text { - Blue } \\ \text { Xylopia sp. (Jangkang) } & \text { - Yellow } \\ \text { Macaranga gigantean (Mahang gajah) } & \text { - Cyan } \\ \text { Ixonanthes icosandra (Pagar anak) } & \text { - Maroon }\end{array}$

Streblus elongates (Tempinis)

Cannarium sp. (Kedondong)

Hevea sp. (Getah)

- Sea Green

- Purple

- Magenta

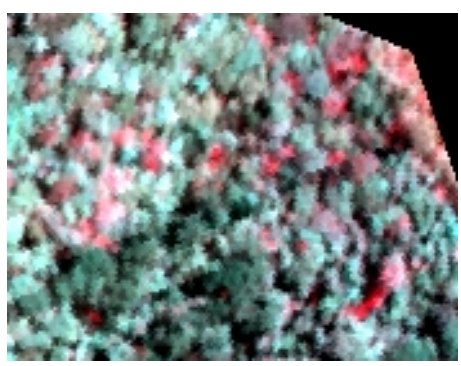

(a)

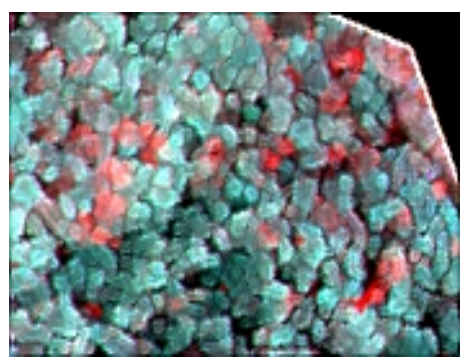

(b)

Fig. 2a: Image of the 2 ha plots in Taman Rimba Ilmu before image enhancement (b) Enhanced image of 2 ha plots in Taman Rimba Ilmu after using a Sobel $3 \times 3$ Filter at $80 \%$

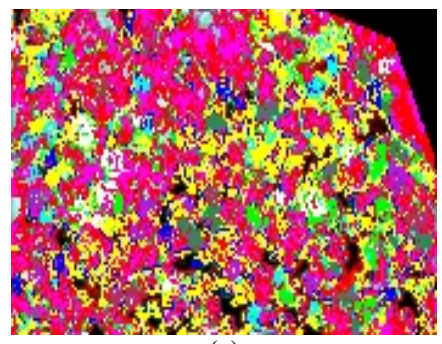

(a)

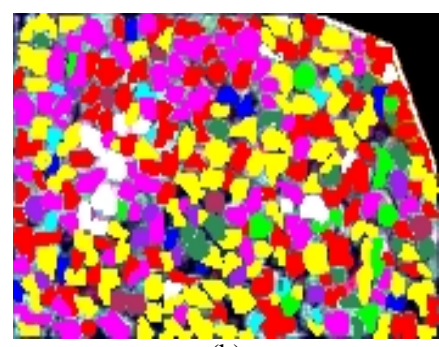

(b)

Fig. 3a: Image classification of 10 tree species using SAM (b) Post classification using clump and sieve method for 10 tree species in study area 
Am. J. Applied Sci., 7 (4): 493-499, 2010

Table 1: The GPS locations of the 297 trees identified, quantified and verified from the UPM-APSB's AISA data in Taman Rimba Ilmu, University Malaya

\begin{tabular}{|c|c|c|c|}
\hline Species name & Latitude & Longitude & $\begin{array}{l}\text { Ground } \\
\text { Verification }\end{array}$ \\
\hline Xylopia sp. & $3^{\circ} 7^{\prime} 43.69^{\prime \prime}$ & 101³9’9.38”' & Confirmed \\
\hline Xylopia sp. & $3^{\circ} 7^{\prime} 43.73^{\prime \prime}$ & 101³9’9.77”' & Confirmed \\
\hline Xylopia sp. & 37’43.65’' & 101³9’12.55” & Confirmed \\
\hline Xylopia sp. & $3^{\circ} 7^{\prime} 43.59^{\prime \prime}$ & 101³9’12.81” & Confirmed \\
\hline Xylopia sp. & 3॰7’43.49’' & 101³9’11.03”' & Confirmed \\
\hline Xylopia sp. & 3०7’43.52', & 101³9’12.42” & Confirmed \\
\hline Xylopia sp. & $3^{\circ} 7^{\prime} 43.49^{\prime \prime}$ & 101³9’13.33”' & Confirmed \\
\hline Xylopia sp. & $3^{\circ} 7^{\prime} 43.27^{\prime \prime}$ & 101³9’9.32” & Confirmed \\
\hline Xylopia sp. & $3^{\circ} 7^{\prime} 43.37^{\prime \prime}$ & 101³9’10.68” & Confirmed \\
\hline Xylopia sp. & 3०7’43.29’' & 101³9’13.17”' & Confirmed \\
\hline Xylopia sp. & $3^{\circ} 7^{\prime} 43.16^{\prime \prime}$ & 101³9’13.46” & Confirmed \\
\hline Xylopia sp. & $3^{\circ} 7^{\prime} 43.10^{\prime \prime}$ & 101³9’12.62” & Confirmed \\
\hline Xylopia sp. & $3^{\circ} 7^{\prime} 42.98^{\prime \prime}$ & 101³9’9.35” & Confirmed \\
\hline Xylopia sp. & $3^{\circ} 7^{\prime} 42.97 ’$ & $101^{\circ} 39^{\prime} 13.23^{\prime \prime}$ & Confirmed \\
\hline Xylopia sp. & $3^{\circ} 7^{\prime} 42.77^{\prime \prime}$ & 101'39'13.33”' & Confirmed \\
\hline Xylopia sp. & 3०7’42.74', & 101³9’12.87”' & Confirmed \\
\hline Xylopia sp. & $3^{\circ} 7^{\prime} 42.77^{\prime \prime}$ & 101'39'12.62" & Confirmed \\
\hline Xylopia sp. & $3^{\circ} 7^{\prime} 42.68 ’$ & 101³9’11.77”' & Confirmed \\
\hline Xylopia sp. & $3^{\circ} 7^{\prime} 42.65^{\prime \prime}$ & 101³9’10.61” & Confirmed \\
\hline Xylopia sp. & $3^{\circ} 7^{\prime} 42.58^{\prime \prime}$ & 101³9’13.72”' & Confirmed \\
\hline Xylopia sp. & $3^{\circ} 7^{\prime} 42.41^{\prime \prime}$ & $101^{\circ} 39 ' 13.46^{\prime \prime}$ & Confirmed \\
\hline Xylopia sp. & $3^{\circ} 7^{\prime} 42.32 \prime$ & $101^{\circ} 39 ' 12.49 ”$ & Confirmed \\
\hline Xylopia sp. & $3^{\circ} 7^{\prime} 42.32^{\prime \prime}$ & 101'39'11.32”" & Confirmed \\
\hline Xylopia sp. & $3^{\circ} 7^{\prime} 42.33^{\prime \prime}$ & $101^{\circ} 39^{\prime} 9.80^{\prime \prime}$ & Confirmed \\
\hline Xylopia sp. & $3^{\circ} 7^{\prime} 42.29^{\prime \prime}$ & $101^{\circ} 39 ' 10.38^{\prime \prime}$ & Confirmed \\
\hline Xylopia sp. & 3॰7’42.23’’ & 101³9’10.77”' & Confirmed \\
\hline Xylopia sp. & 3०7’42.19’' & $101^{\circ} 39^{\prime} 11.74 ”$ & Confirmed \\
\hline Xylopia sp. & 3०7’42.22'’ & 101³9’13.13”' & Confirmed \\
\hline Xylopia sp. & 3०7’42.06’' & 101³9’13.26”" & Confirmed \\
\hline Xylopia sp. & $3^{\circ} 7^{\prime} 42.06^{\prime \prime}$ & 101³9’12.52” & Confirmed \\
\hline Xylopia sp. & $3^{\circ} 7^{\prime} 42.06^{\prime \prime}$ & $101^{\circ} 39^{\prime} 12.26^{\prime \prime}$ & Confirmed \\
\hline Xylopia sp. & $3^{\circ} 7^{\prime} 42.13^{\prime \prime}$ & $101^{\circ} 39^{\prime} 9.41^{\prime \prime}$ & Confirmed \\
\hline Xylopia sp. & $3^{\circ} 7^{\prime} 41.94 \prime$ & $101^{\circ} 39^{\prime} 9.67^{\prime \prime}$ & Confirmed \\
\hline Xylopia sp. & $3^{\circ} 7^{\prime} 41.99^{\prime \prime}$ & 101'39'14.39”' & Confirmed \\
\hline Xylopia sp. & $3^{\circ} 7^{\prime} 41.90^{\prime \prime}$ & $101^{\circ} 39^{\prime} 11.67^{\prime \prime}$ & Confirmed \\
\hline Xylopia sp. & 3॰7’41.83’' & 101'39'11.38”' & Confirmed \\
\hline Xylopia sp. & 3०7’41.83’' & $101^{\circ} 39^{\prime} 13.00^{\prime \prime}$ & Confirmed \\
\hline Xylopia sp. & $3^{\circ} 7^{\prime} 41.77^{\prime \prime}$ & 101'39'12.03”' & Confirmed \\
\hline Xylopia sp. & $3^{\circ} 7^{\prime} 41.79^{\prime \prime}$ & $101^{\circ} 39^{\prime} 14.56^{\prime \prime}$ & Confirmed \\
\hline Xylopia sp. & $3^{\circ} 7^{\prime} 41.51^{\prime \prime}$ & 101³9’11.16” & Confirmed \\
\hline Xylopia sp. & $3^{\circ} 7^{\prime} 41.51^{\prime \prime}$ & 101³9’12.48”' & Confirmed \\
\hline Xylopia sp. & $3^{\circ} 7^{\prime} 41.44^{\prime \prime}$ & $101^{\circ} 39^{\prime} 11.51^{\prime \prime}$ & Confirmed \\
\hline Xylopia sp. & $3^{\circ} 7^{\prime} 41.28^{\prime \prime}$ & $101^{\circ} 39 ' 10.41^{\prime \prime}$ & Confirmed \\
\hline Xylopia sp. & $3^{\circ} 7^{\prime} 41.31^{\prime \prime}$ & $101^{\circ} 399^{\prime} 13.68^{\prime \prime}$ & Confirmed \\
\hline Xylopia sp. & $3^{\circ} 7^{\prime} 41.18 ’$ & $101^{\circ} 39 ’ 11.93^{\prime \prime}$ & Confirmed \\
\hline Xylopia sp. & $3^{\circ} 7^{\prime} 41.15^{\prime \prime}$ & $101^{\circ} 39^{\prime} 9.96^{\prime \prime}$ & Confirmed \\
\hline Xylopia sp. & $3^{\circ} 7^{\prime} 41.06^{\prime \prime}$ & $101^{\circ} 39^{\prime} 10.48^{\prime \prime}$ & Confirmed \\
\hline Xylopia sp. & $3^{\circ} 7^{\prime} 41.05^{\prime}$ & $101^{\circ} 39^{\prime} 12.35^{\prime \prime}$ & Confirmed \\
\hline Xylopia sp. & $3^{\circ} 7^{\prime} 41.02 \prime$ & $101^{\circ} 39^{\prime} 12.58^{\prime \prime}$ & Confirmed \\
\hline Xylopia sp. & $3^{\circ} 7^{\prime} 41.02$ ', & 101'39'13.71” & Confirmed \\
\hline Xylopia sp. & $3^{\circ} 7^{\prime} 41.01^{\prime \prime}$ & 101³9’14.26” & Confirmed \\
\hline Xylopia sp. & $3^{\circ} 7^{\prime} 40.98^{\prime \prime}$ & 101³9’13.03”' & Confirmed \\
\hline Xylopia sp. & 37’40.99’' & 101³9’12.58”' & Confirmed \\
\hline Xylopia sp. & $3^{\circ} 7^{\prime} 40.96^{\prime \prime}$ & $101^{\circ} 39^{\prime} 9.47^{\prime \prime}$ & Confirmed \\
\hline Xylopia sp. & $3^{\circ} 7^{\prime} 40.96 \prime$ & $101^{\circ} 39^{\prime} 10.05^{\prime \prime}$ & Confirmed \\
\hline Xylopia sp. & $3^{\circ} 7^{\prime} 40.92 \prime$ & 101'39'11.09” & Confirmed \\
\hline Xylopia sp. & $3^{\circ} 7^{\prime} 40.96 \prime$ & $101^{\circ} 39^{\prime} 11.67^{\prime \prime}$ & Confirmed \\
\hline Xylopia sp. & $3^{\circ} 7^{\prime} 40.92^{\prime \prime}$ & 101'39'11.93”" & Confirmed \\
\hline Xylopia sp. & $3^{\circ} 7^{\prime} 40.86^{\prime \prime}$ & $101^{\circ} 39^{\prime} 12.22^{\prime \prime}$ & Confirmed \\
\hline Xylopia sp. & $3^{\circ} 7^{\prime} 40.73^{\prime \prime}$ & $101^{\circ} 39^{\prime} 9.86^{\prime \prime}$ & Confirmed \\
\hline Xylopia sp. & $3^{\circ} 7^{\prime} 40.73^{\prime \prime}$ & 101³9’10.31” & Confirmed \\
\hline Xylopia sp. & 37’40.82’' & 101³9’14.49”' & Confirmed \\
\hline Xylopia sp. & 3०7’40.72', & 101³9’13.71” & Confirmed \\
\hline Xylopia sp. & $3^{\circ} 7^{\prime} 40.63^{\prime \prime}$ & $101^{\circ} 39 ' 10.89 ”$ & Confirmed \\
\hline Xylopia sp. & $3^{\circ} 7^{\prime} 40.66^{\prime \prime}$ & $101^{\circ} 39 ' 14.13 ”$ & Confirmed \\
\hline Xylopia sp. & $3^{\circ} 7^{\prime} 40.63^{\prime \prime}$ & 101³9’11.74”' & Confirmed \\
\hline Xylopia sp. & $3^{\circ} 7^{\prime} 40.56^{\prime \prime}$ & 101³9’12.80” & Confirmed \\
\hline Xylopia sp. & $3^{\circ} 7^{\prime} 40.56^{\prime \prime}$ & $101^{\circ} 39 ' 14.42 \prime$ & Confirmed \\
\hline Xylopia sp. & $3^{\circ} 7^{\prime} 40.53^{\prime \prime}$ & $101^{\circ} 39 ' 11.41^{\prime \prime}$ & Confirmed \\
\hline Xylopia sp. & $3^{\circ} 7^{\prime} 40.47^{\prime \prime}$ & 101³9’12.09”' & Confirmed \\
\hline Xylopia sp. & $3^{\circ} 7^{\prime} 40.50 \prime$ & $101^{\circ} 39^{\prime} 12.51^{\prime \prime}$ & Confirmed \\
\hline Xylopia sp. & $3^{\circ} 7^{\prime} 40.44^{\prime \prime}$ & 101'39'11.09” & Confirmed \\
\hline
\end{tabular}

Table 1: Continued

\begin{tabular}{|c|c|c|c|}
\hline Xylopia sp. & $3^{\circ} 7^{\prime} 40.44^{\prime \prime}$ & 101'39’9.99”' & Confirmed \\
\hline Xylopia sp. & $3^{\circ} 7^{\prime} 40.37^{\prime \prime}$ & $101^{\circ} 39^{\prime} 10.89 ”$ & Confirmed \\
\hline Xylopia sp. & $3^{\circ} 7^{\prime} 40.15^{\prime \prime}$ & 101³9’9.24” & Confirmed \\
\hline Xylopia sp. & $3^{\circ} 7^{\prime} 40.14^{\prime \prime}$ & $101^{\circ} 39^{\prime} 10.76^{\prime \prime}$ & Confirmed \\
\hline Xylopia sp. & $3^{\circ} 7^{\prime} 40.14^{\prime \prime}$ & $101^{\circ} 39 ' 11.15^{\prime \prime}$ & Confirmed \\
\hline Xylopia sp. & $3^{\circ} 7^{\prime} 40.17^{\prime \prime}$ & 101'39'11.54”' & Confirmed \\
\hline Xylopia sp. & $3^{\circ} 7^{\prime} 40.11^{\prime \prime}$ & $101^{\circ} 39 ' 11.86^{\prime \prime}$ & Confirmed \\
\hline Xylopia sp. & $3^{\circ} 7^{\prime} 40.08^{\prime \prime}$ & 101³9'12.12” & Confirmed \\
\hline Xylopia sp. & $3^{\circ} 7^{\prime} 40.14^{\prime \prime}$ & 101³9'13.06”" & Confirmed \\
\hline Xylopia sp. & $3^{\circ} 7^{\prime} 40.14^{\prime \prime}$ & 101³9’13.94”" & Confirmed \\
\hline Xylopia sp. & $3^{\circ} 7^{\prime} 40.10^{\prime \prime}$ & 101³9’14.49”' & Confirmed \\
\hline Hevea sp. & $3^{\circ} 7^{\prime} 43.69^{\prime \prime}$ & 101³9’10.81" & Confirmed \\
\hline Hevea sp. & $3^{\circ} 7^{\prime} 43.59^{\prime \prime}$ & 101³9’10.09”' & Confirmed \\
\hline Hevea sp. & $3^{\circ} 7^{\prime} 43.47^{\prime \prime}$ & 101³9’9.83”' & Confirmed \\
\hline Hevea sp. & $3^{\circ} 7^{\prime} 43.43^{\prime \prime}$ & $101^{\circ} 39^{\prime} 11.26^{\prime \prime}$ & Confirmed \\
\hline Hevea sp. & $3^{\circ} 7^{\prime} 43.43^{\prime \prime}$ & 101³9’11.45” & Confirmed \\
\hline Hevea sp. & $3^{\circ} 7^{\prime} 43.49^{\prime \prime}$ & 101³9’11.84”' & Confirmed \\
\hline Hevea sp. & $3^{\circ} 7^{\prime} 43.46^{\prime \prime}$ & 101³9'12.16”' & Confirmed \\
\hline Hevea sp. & $3^{\circ} 7^{\prime} 43.42^{\prime \prime}$ & 101'39'13.59”' & Confirmed \\
\hline Hevea sp. & $3^{\circ} 7^{\prime} 43.33^{\prime \prime}$ & 101³9’10.48”' & Confirmed \\
\hline Hevea sp. & $3^{\circ} 7^{\prime} 43.30^{\prime \prime}$ & 101³9’11.94”" & Confirmed \\
\hline Hevea sp. & $3^{\circ} 7^{\prime} 43.17^{\prime \prime}$ & 101³9’11.19” & Confirmed \\
\hline Hevea sp. & $3^{\circ} 7^{\prime} 43.07^{\prime \prime}$ & 101³9’10.97” & Confirmed \\
\hline Hevea sp. & $3^{\circ} 7^{\prime} 43.17^{\prime \prime}$ & 101³9’12.36”' & Confirmed \\
\hline Hevea sp. & $3^{\circ} 7^{\prime} 43.16^{\prime \prime}$ & 101³9’13.62”' & Confirmed \\
\hline Hevea sp. & $3^{\circ} 7^{\prime} 42.97^{\prime \prime}$ & $101^{\circ} 39^{\prime} 12.45^{\prime \prime}$ & Confirmed \\
\hline Hevea sp. & $3^{\circ} 7^{\prime} 42.88^{\prime \prime}$ & $101^{\circ} 39^{\prime} 11.16^{\prime \prime}$ & Confirmed \\
\hline Hevea sp. & $3^{\circ} 7^{\prime} 42.84^{\prime \prime}$ & 101³9’11.52” & Confirmed \\
\hline Hevea sp. & $3^{\circ} 7^{\prime} 42.78^{\prime \prime}$ & 101³9’12.42”' & Confirmed \\
\hline Hevea sp. & $3^{\circ} 7^{\prime} 42.65^{\prime \prime}$ & 101³9’11.26”' & Confirmed \\
\hline Hevea sp. & $3^{\circ} 7^{\prime} 42.71^{\prime \prime}$ & 101³9’10.84” & Confirmed \\
\hline Hevea sp. & $3^{\circ} 7^{\prime} 42.51^{\prime \prime}$ & $101^{\circ} 39^{\prime} 12.68^{\prime \prime}$ & Confirmed \\
\hline Hevea sp. & $3^{\circ} 7^{\prime} 43.21^{\prime \prime}$ & $101^{\circ} 399^{\prime} 9.61^{\prime \prime}$ & Confirmed \\
\hline Hevea sp. & $3^{\circ} 7^{\prime} 43.11^{\prime \prime}$ & $101^{\circ} 39^{\prime} 9.90^{\prime \prime}$ & Confirmed \\
\hline Hevea sp. & $3^{\circ} 7^{\prime} 42.69^{\prime \prime}$ & $101^{\circ} 39^{\prime} 9.18^{\prime \prime}$ & Confirmed \\
\hline Hevea sp. & $3^{\circ} 7^{\prime} 42.75^{\prime \prime}$ & $101^{\circ} 39^{\prime} 9.44^{\prime \prime}$ & Confirmed \\
\hline Hevea sp. & $3^{\circ} 7^{\prime} 42.72^{\prime \prime}$ & 101³9’9.67”' & Confirmed \\
\hline Hevea sp. & $3^{\circ} 7^{\prime} 42.52^{\prime \prime}$ & 101³9’9.67”' & Confirmed \\
\hline Hevea sp. & $3^{\circ} 7^{\prime} 42.36^{\prime \prime}$ & $101^{\circ} 39^{\prime} 10.28 ”$ & Confirmed \\
\hline Hevea sp. & $3^{\circ} 7^{\prime} 42.22^{\prime \prime}$ & 101³9'12.87”' & Confirmed \\
\hline Hevea sp. & $3^{\circ} 7^{\prime} 41.97^{\prime \prime}$ & 101³9’9.18” & Confirmed \\
\hline Hevea sp. & $3^{\circ} 7^{\prime} 41.87^{\prime \prime}$ & 101³9’10.02” & Confirmed \\
\hline Hevea sp. & $3^{\circ} 7^{\prime} 41.84^{\prime \prime}$ & 101³9’10.80” & Confirmed \\
\hline Hevea sp. & $3^{\circ} 7^{\prime} 41.74^{\prime \prime}$ & 101³9’10.54”' & Confirmed \\
\hline Hevea sp. & $3^{\circ} 7^{\prime} 41.61^{\prime \prime}$ & $101^{\circ} 39^{\prime} 9.54^{\prime \prime}$ & Confirmed \\
\hline Hevea sp. & $3^{\circ} 7^{\prime} 41.51^{\prime \prime}$ & $101^{\circ} 39 ' 10.64 ”$ & Confirmed \\
\hline Hevea sp. & $3^{\circ} 7^{\prime} 41.42^{\prime \prime}$ & $101^{\circ} 39^{\prime} 9.76^{\prime \prime}$ & Confirmed \\
\hline Hevea sp. & $3^{\circ} 7^{\prime} 41.31^{\prime \prime}$ & 101³9’10.93”' & Confirmed \\
\hline Hevea sp. & $3^{\circ} 7^{\prime} 41.61^{\prime \prime}$ & 101³9’11.29” & Confirmed \\
\hline Hevea sp. & $3^{\circ} 7^{\prime} 40.99^{\prime \prime}$ & $101^{\circ} 39^{\prime} 10.28 ”$ & Confirmed \\
\hline Hevea sp. & $3^{\circ} 7^{\prime} 40.79^{\prime \prime}$ & 101³9’10.73”' & Confirmed \\
\hline Hevea sp. & $3^{\circ} 7^{\prime} 40.90^{\prime \prime}$ & $101^{\circ} 39^{\prime} 9.12^{\prime \prime}$ & Confirmed \\
\hline Hevea sp. & $3^{\circ} 7^{\prime} 40.41^{\prime \prime}$ & 101³9’9.66”' & Confirmed \\
\hline Hevea sp. & $3^{\circ} 7^{\prime} 40.28^{\prime \prime}$ & $101^{\circ} 39^{\prime} 9.24^{\prime \prime}$ & Confirmed \\
\hline Hevea sp. & $3^{\circ} 7^{\prime} 40.15^{\prime \prime}$ & $101^{\circ} 39^{\prime} 9.76^{\prime \prime}$ & Confirmed \\
\hline Hevea sp. & $3^{\circ} 7^{\prime} 40.18^{\prime \prime}$ & 101³9’10.38”' & Confirmed \\
\hline Hevea sp. & $3^{\circ} 7^{\prime} 40.31^{\prime \prime}$ & 101³9’11.25” & Confirmed \\
\hline Hevea sp. & $3^{\circ} 7^{\prime} 41.47^{\prime \prime}$ & 101³9’12.00”' & Confirmed \\
\hline Hevea sp. & $3^{\circ} 7^{\prime} 41.60^{\prime \prime}$ & 101³9’12.22”" & Confirmed \\
\hline Hevea sp. & $3^{\circ} 7^{\prime} 41.73^{\prime \prime}$ & 101³9’12.74”' & Confirmed \\
\hline Hevea sp. & $3^{\circ} 7^{\prime} 41.25^{\prime \prime}$ & 101³9’12.84” & Confirmed \\
\hline Hevea sp. & $3^{\circ} 7^{\prime} 41.05^{\prime \prime}$ & 101³9’12.13”' & Confirmed \\
\hline Hevea sp. & $3^{\circ} 7^{\prime} 41.57^{\prime \prime}$ & 101³9’13.78”' & Confirmed \\
\hline Hevea sp. & $3^{\circ} 7^{\prime} 41.57^{\prime \prime}$ & 101³9’14.30”' & Confirmed \\
\hline Hevea sp. & $3^{\circ} 7^{\prime} 40.72^{\prime \prime}$ & 101³9’13.97”' & Confirmed \\
\hline Hevea sp. & $3^{\circ} 7^{\prime} 40.53^{\prime \prime}$ & 101³9’13.87” & Confirmed \\
\hline Hevea sp. & $3^{\circ} 7^{\prime} 40.20^{\prime \prime}$ & 101³9’13.32” & Confirmed \\
\hline Ixonanthes sp. & $3^{\circ} 7^{\prime} 43.04^{\prime \prime}$ & $101^{\circ} 39^{\prime} 9.09^{\prime \prime}$ & Confirmed \\
\hline Ixonanthes sp. & $3^{\circ} 7^{\prime} 42.23^{\prime \prime}$ & 101³9’9.15” & Confirmed \\
\hline Ixonanthes sp. & $3^{\circ} 7^{\prime} 42.13^{\prime \prime}$ & 101³9’9.93” & Confirmed \\
\hline Ixonanthes sp. & $3^{\circ} 7^{\prime} 41.90^{\prime \prime}$ & 101³9’9.34” & Confirmed \\
\hline Ixonanthes sp. & $3^{\circ} 7^{\prime} 41.74^{\prime \prime}$ & 101³9’9.12” & Confirmed \\
\hline Ixonanthes sp. & $3^{\circ} 7^{\prime} 41.68^{\prime \prime}$ & $101^{\circ} 39^{\prime} 9.80^{\prime \prime}$ & Confirmed \\
\hline Ixonanthes sp. & $3^{\circ} 7^{\prime} 41.45^{\prime \prime}$ & $101^{\circ} 39^{\prime} 9.12^{\prime \prime}$ & Confirmed \\
\hline Ixonanthes sp. & $3^{\circ} 7^{\prime} 41.16^{\prime \prime}$ & $101^{\circ} 39^{\prime} 9.18^{\prime \prime}$ & Confirmed \\
\hline Ixonanthes sp. & $3^{\circ} 7^{\prime} 41.19^{\prime \prime}$ & $101^{\circ} 39^{\prime} 9.47^{\prime \prime}$ & Confirmed \\
\hline Ixonanthes sp. & $3^{\circ} 7^{\prime} 41.12^{\prime \prime}$ & $101^{\circ} 39^{\prime} 10.22^{\prime \prime}$ & $\begin{array}{l}\text { Misclassified as } \\
\text { Ixonanthes icosandra }\end{array}$ \\
\hline
\end{tabular}


Am. J. Applied Sci., 7 (4): 493-499, 2010

Table 1: Continued

\begin{tabular}{|c|c|c|c|}
\hline Ixonanthes sp. & $3^{\circ} 7^{\prime} 40.96^{\prime \prime}$ & $101^{\circ} 39^{\prime} 9.86^{\prime \prime}$ & Confirmed \\
\hline Ixonanthes sp. & $3^{\circ} 7^{\prime} 40.99 ’$ & 101³9’10.90” & Confirmed \\
\hline Ixonanthes sp. & $3^{\circ} 7^{\prime} 40.89 ’$ & 101³9’10.60” & Confirmed \\
\hline Ixonanthes sp. & $3^{\circ} 7^{\prime} 40.76^{\prime \prime}$ & 101³9’10.09”' & Confirmed \\
\hline Ixonanthes sp. & $3^{\circ} 7^{\prime} 40.76^{\prime \prime}$ & 101³9’11.22” & Confirmed \\
\hline Ixonanthes sp. & $3^{\circ} 7^{\prime} 40.54^{\prime \prime}$ & $101^{\circ} 39^{\prime} 9.50^{\prime \prime}$ & Confirmed \\
\hline Ixonanthes sp. & $3^{\circ} 7^{\prime} 40.57^{\prime \prime}$ & $101^{\circ} 39^{\prime} 9.73^{\prime \prime}$ & Confirmed \\
\hline Ixonanthes sp. & $3^{\circ} 7^{\prime} 40.18^{\prime \prime}$ & 101³9’10.02” & Confirmed \\
\hline Ixonanthes sp. & $3^{\circ} 7^{\prime} 40.34^{\prime \prime}$ & 101³9’11.77” & Confirmed \\
\hline Ixonanthes sp. & $3^{\circ} 7^{\prime} 43.63^{\prime \prime}$ & 101³9’10.42” & Confirmed \\
\hline Ixonanthes sp. & $3^{\circ} 7^{\prime} 43.62^{\prime \prime}$ & 101³9’11.00” & Confirmed \\
\hline Ixonanthes sp. & $3^{\circ} 7^{\prime} 43.24^{\prime \prime}$ & 101³9’10.06” & Confirmed \\
\hline Ixonanthes sp. & $3^{\circ} 7^{\prime} 43.14^{\prime \prime}$ & 101³9’10.29” & Confirmed \\
\hline Ixonanthes sp. & $3^{\circ} 7^{\prime} 43.14^{\prime \prime}$ & 101³9’10.67”' & Confirmed \\
\hline Ixonanthes sp. & $3^{\circ} 7^{\prime} 42.85^{\prime \prime}$ & 101³9’10.06” & $\begin{array}{l}\text { Misclassified as } \\
\text { Ixonanthes icosandra }\end{array}$ \\
\hline Ixonanthes sp. & $3^{\circ} 7^{\prime} 42.81^{\prime \prime}$ & 101³9’10.48”' & Confirmed \\
\hline Ixonanthes sp. & $3^{\circ} 7^{\prime} 42.65^{\prime \prime}$ & 101³9’10.06” & Confirmed \\
\hline Ixonanthes sp. & $3^{\circ} 7^{\prime} 42.55^{\prime \prime}$ & 101³9’10.25” & Confirmed \\
\hline Ixonanthes sp. & $3^{\circ} 7^{\prime} 42.42^{\prime \prime}$ & 101³9’11.06” & Confirmed \\
\hline Ixonanthes sp. & $3^{\circ} 7^{\prime} 42.29^{\prime \prime}$ & 101³9’10.54” & Confirmed \\
\hline Ixonanthes sp. & $3^{\circ} 7^{\prime} 42.06^{\prime \prime}$ & 101³9’11.09” & Confirmed \\
\hline Ixonanthes sp. & $3^{\circ} 7^{\prime} 42.03^{\prime \prime}$ & 101³9’10.83”' & Confirmed \\
\hline Ixonanthes sp. & $3^{\circ} 7^{\prime} 41.74^{\prime \prime}$ & 101³9’11.06”' & Confirmed \\
\hline Ixonanthes sp. & $3^{\circ} 7^{\prime} 42.68^{\prime \prime}$ & 101³9’11.03” & Confirmed \\
\hline Ixonanthes sp. & $3^{\circ} 7^{\prime} 43.62^{\prime \prime}$ & 101³9’11.39” & Confirmed \\
\hline Ixonanthes sp. & $3^{\circ} 7^{\prime} 43.07^{\prime \prime}$ & 101³9’11.42” & Confirmed \\
\hline Ixonanthes sp. & $3^{\circ} 7^{\prime} 42.58^{\prime \prime}$ & 101³9’11.48”' & Confirmed \\
\hline Ixonanthes sp. & $3^{\circ} 7^{\prime} 42.09^{\prime \prime}$ & 101³9’11.42” & Confirmed \\
\hline Ixonanthes sp. & $3^{\circ} 7^{\prime} 43.65^{\prime \prime}$ & 101³9’11.91” & Confirmed \\
\hline Ixonanthes sp. & $3^{\circ} 7^{\prime} 43.69^{\prime \prime}$ & 101³9’12.23” & Confirmed \\
\hline Ixonanthes sp. & $3^{\circ} 7^{\prime} 43.33^{\prime \prime}$ & 101³9’11.65” & Confirmed \\
\hline Ixonanthes sp. & $3^{\circ} 7^{\prime} 43.13^{\prime \prime}$ & 101³9’11.74” & Confirmed \\
\hline Ixonanthes sp. & $3^{\circ} 7^{\prime} 43.10^{\prime \prime}$ & 101³9’12.07”' & Confirmed \\
\hline Ixonanthes sp. & $3^{\circ} 7^{\prime} 42.13^{\prime \prime}$ & 101³9’12.03”' & Confirmed \\
\hline Ixonanthes sp. & $3^{\circ} 7^{\prime} 41.87^{\prime \prime}$ & 101³9’11.87”' & Confirmed \\
\hline Ixonanthes sp. & $3^{\circ} 7^{\prime} 41.31^{\prime \prime}$ & 101³9’12.09” & Confirmed \\
\hline Ixonanthes sp. & $3^{\circ} 7^{\prime} 41.73^{\prime \prime}$ & 101³9’12.42” & Confirmed \\
\hline Ixonanthes sp. & $3^{\circ} 7^{\prime} 41.18^{\prime \prime}$ & 101³9’12.55” & Confirmed \\
\hline Ixonanthes sp. & $3^{\circ} 7^{\prime} 40.66^{\prime \prime}$ & 101³9’12.35” & Confirmed \\
\hline Ixonanthes sp. & $3^{\circ} 7^{\prime} 40.34^{\prime \prime}$ & 101³9’12.42” & Confirmed \\
\hline Ixonanthes sp. & $3^{\circ} 7^{\prime} 40.92^{\prime \prime}$ & 101³9’12.77”' & Confirmed \\
\hline Ixonanthes sp. & $3^{\circ} 7^{\prime} 43.39^{\prime \prime}$ & 101³9’12.68”' & Confirmed \\
\hline Ixonanthes sp. & $3^{\circ} 7^{\prime} 43.39^{\prime \prime}$ & 101³9’12.91” & Confirmed \\
\hline Ixonanthes sp. & $3^{\circ} 7^{\prime} 43.65^{\prime \prime}$ & 101³9’13.01” & Confirmed \\
\hline Ixonanthes sp. & $3^{\circ} 7^{\prime} 42.45^{\prime \prime}$ & 101³9’12.84” & Confirmed \\
\hline Ixonanthes sp. & $3^{\circ} 7^{\prime} 42.48^{\prime \prime}$ & 101³9’13.10” & Confirmed \\
\hline Ixonanthes sp. & $3^{\circ} 7^{\prime} 41.99^{\prime \prime}$ & 101³9’12.81” & Confirmed \\
\hline Ixonanthes sp. & $3^{\circ} 7^{\prime} 42.94^{\prime \prime}$ & 101³9’13.49” & Confirmed \\
\hline Ixonanthes sp. & $3^{\circ} 7^{\prime} 42.77^{\prime \prime}$ & 101³9’13.62” & Confirmed \\
\hline Ixonanthes sp. & $3^{\circ} 7^{\prime} 42.22^{\prime \prime}$ & 101³9’13.71” & Confirmed \\
\hline Ixonanthes sp. & $3^{\circ} 7^{\prime} 41.93^{\prime \prime}$ & 101³9’13.65” & Confirmed \\
\hline Ixonanthes sp. & $3^{\circ} 7^{\prime} 41.80^{\prime \prime}$ & 101³9’13.45” & Confirmed \\
\hline Ixonanthes sp. & $3^{\circ} 7^{\prime} 41.67^{\prime \prime}$ & 101³9’13.26”' & Confirmed \\
\hline Ixonanthes sp. & $3^{\circ} 7^{\prime} 41.50^{\prime \prime}$ & 101³9’13.55” & Confirmed \\
\hline Ixonanthes sp. & $3^{\circ} 7^{\prime} 41.08^{\prime \prime}$ & 101³9’13.36”' & Confirmed \\
\hline Ixonanthes sp. & $3^{\circ} 7^{\prime} 40.72^{\prime \prime}$ & 101³9’13.19” & Confirmed \\
\hline Ixonanthes sp. & $3^{\circ} 7^{\prime} 40.53^{\prime \prime}$ & 101³9’13.26” & Confirmed \\
\hline Ixonanthes sp. & $3^{\circ} 7^{\prime} 43.39^{\prime \prime}$ & 101³9’13.78”' & Confirmed \\
\hline Ixonanthes sp. & $3^{\circ} 7^{\prime} 43.19^{\prime \prime}$ & 101³9’13.91” & Confirmed \\
\hline Ixonanthes sp. & $3^{\circ} 7^{\prime} 42.90^{\prime \prime}$ & 101³9’14.14” & Confirmed \\
\hline Ixonanthes sp. & $3^{\circ} 7^{\prime} 42.64^{\prime \prime}$ & 101³9’14.20” & Confirmed \\
\hline Ixonanthes sp. & $3^{\circ} 7^{\prime} 42.45^{\prime \prime}$ & 101³9’14.27”' & Confirmed \\
\hline Ixonanthes sp. & $3^{\circ} 7^{\prime} 42.25^{\prime \prime}$ & 101³9’14.43” & Confirmed \\
\hline Ixonanthes sp. & $3^{\circ} 7^{\prime} 41.76^{\prime \prime}$ & 101³9’14.36”' & Confirmed \\
\hline Ixonanthes sp. & $3^{\circ} 7^{\prime} 41.50^{\prime \prime}$ & 101³9’14.55” & Confirmed \\
\hline Ixonanthes sp. & $3^{\circ} 7^{\prime} 41.31^{\prime \prime}$ & 101³9’14.20” & Confirmed \\
\hline Ixonanthes sp. & $3^{\circ} 7^{\prime} 40.95^{\prime \prime}$ & 101³9’14.00” & Confirmed \\
\hline Ixonanthes sp. & $3^{\circ} 7^{\prime} 40.85^{\prime \prime}$ & $101^{\circ} 39^{\prime} 14.29 ”$ & Confirmed \\
\hline Ixonanthes sp. & $3^{\circ} 7^{\prime} 40.39^{\prime \prime}$ & $101^{\circ} 39^{\prime} 14.58^{\prime \prime}$ & Confirmed \\
\hline Endospermum diadenum & $3^{\circ} 7^{\prime} 43.20^{\prime \prime}$ & 101³9’9.87” & Confirmed \\
\hline Endospermum diadenum & $3^{\circ} 7^{\prime} 43.07^{\prime \prime}$ & $101^{\circ} 39^{\prime} 12.91^{\prime \prime}$ & Confirmed \\
\hline Endospermum diadenum & $3^{\circ} 7^{\prime} 42.74^{\prime \prime}$ & $101^{\circ} 39^{\prime} 14.04^{\prime \prime}$ & Confirmed \\
\hline Endospermum diadenum & $3^{\circ} 7^{\prime} 42.02^{\prime \prime}$ & $101^{\circ} 39^{\prime} 13.94^{\prime \prime}$ & Confirmed \\
\hline Endospermum diadenum & $3^{\circ} 7^{\prime} 41.51^{\prime \prime}$ & $101^{\circ} 39^{\prime} 10.48^{\prime \prime}$ & Confirmed \\
\hline Endospermum diadenum & $3^{\circ} 7^{\prime} 41.34^{\prime \prime}$ & $101^{\circ} 39^{\prime} 12.29^{\prime \prime}$ & Confirmed \\
\hline Endospermum diadenum & $3^{\circ} 7^{\prime} 41.44^{\prime \prime}$ & 101³9’13.32” & Confirmed \\
\hline
\end{tabular}

Table 1: Continued

Endospermum diadenum $\quad 3^{\circ} 7^{\prime} 41.31^{\prime \prime} \quad 101^{\circ} 39^{\prime} 13.07^{\prime \prime} \quad$ Confirmed

Endospermum diadenum $3^{\circ} 7^{\prime} 40.98$ ', 101³9'13.84" Confirmed

Endospermum diadenum $\quad 3^{\circ} 7^{\prime} 40.43^{\prime}$ ' 101'39'12.71" Confirmed

Endospermum diadenum $\quad 3^{\circ} 7^{\prime} 40.30^{\prime \prime} \quad 1^{\circ} 1^{\circ} 39^{\prime} 13.77^{\prime \prime} \quad$ Confirmed

Pellacalyx sp.

$3^{\circ} 7^{\prime} 43.72$ ', $101^{\circ} 39^{\prime} 12.62^{\prime \prime}$ Confirmed

Pellacalyx sp.

Pellacalyx sp.

Pellacalyx sp.

37'43.33', 101'39'12.39'

3०7'43.10', 101'39'14.01"'

3०7'42.52', 101'39'10.45',

$3^{\circ} 7^{\prime} 42.49^{\prime \prime} \quad 101^{\circ} 39^{\prime} 10.74 \prime$

$3^{\circ} 7^{\prime} 42.36^{\prime \prime}, 101^{\circ} 39^{\prime} 10.09^{\prime \prime}$

3०7'42.03', 101'39'10.38',

$3^{\circ} 7^{\prime} 42.06^{\prime \prime} \quad 101^{\circ} 39^{\prime} 13.46^{\prime \prime}$

3०7'41.64', 101'39'10.25'

$3^{\circ} 7^{\prime} 41.45^{\prime \prime} \quad 101^{\circ} 39^{\prime} 9.99^{\prime \prime}$

3०7'41.32', 101'39'10.18',

$3^{\circ} 7^{\prime} 41.57^{\prime \prime} \quad 101^{\circ} 39^{\prime} 12.68^{\prime \prime}$

37'41.54', 101³9'13.03'”

$3^{\circ} 7^{\prime} 40.60^{\prime \prime} \quad 101^{\circ} 39^{\prime} 11.12^{\prime \prime}$

3०7'43.50', 101'39'9.41"

37'43.00', 101'39'13.75"

37'42.58', 101³9'12.45"

3०7'42.45', 101'39'11.74'

3०7’42.58', 101'39'14.04"

3ำ42.15', 101'39'14.17',

3ํ'41.89', 101'39'13.29'

Confirmed

Confirmed

Confirmed

Confirmed

Confirmed

Confirmed

Confirmed

Confirmed

Confirmed

Confirmed

Confirmed

Confirmed

Confirmed

Confirmed

Confirmed

Confirmed

Confirmed

Confirmed

Confirmed

Confirmed

$\begin{array}{lr}\text { Streblus elongates } & 3^{\circ} 7^{\prime} 41.89^{\prime} \\ \text { Shortcut to JForOX2 NimaKJ.Lnk }\end{array}$

Streblus elongates

3०7'41.35', 101³9'11.71”

Streblus elongates

Streblus elongates

Streblus elongates

$3^{\circ} 7^{\prime} 41$

3०7'41.09', 101'39'11.48'

3०7’40.76', 101'39'13.36"

37'40.46”, 10139'13.52"

$3^{\circ} 7^{\prime} 40.17$ ', $101^{\circ} 39$ '13.61',

3०7'41.11', 101'39'14.13"

3०7'41.08', 101³9'14.52"

3०7'42.91'” 101'39'9.64"

3०7'42.91', 101³9'10.77”,

3०7'43.10', 101³9'13.33”,

3ㄱ'42 87',

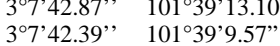

3०7'42.39', 101'39'10.87',

3०7'41.38', 101'39'9.54"

$3^{\circ} 7^{\prime} 40.66^{\prime \prime} \quad 101^{\circ} 39^{\prime} 12.58^{\prime \prime}$

3०7'40.24'” 101'39'12.25',

$3^{\circ} 7^{\prime} 40.27^{\prime \prime} \quad 101^{\circ} 39^{\prime} 13.06^{\prime \prime}$

37'40.46', 101'39'13.52"

37'43.63', 101'39'10.61'

$3^{\circ} 7^{\prime} 42.84$ ', $101^{\circ} 39^{\prime} 11.87^{\prime \prime}$

3०7'42.71', 101'39'12.13"

37'42.80", 101³9'13.91"

3०7'42.03', 101'39'9.57'”

3ํ'40.57', 101'39'9.08”

3०7'40.86', 101³9'9.67',

3०7'41.18', 101'39'11.12',

$3^{\circ} 7^{\prime} 40.83^{\prime}, \quad 101^{\circ} 39^{\prime} 11.54$ '

Cratoxylum sp.

Cratoxylum sp.

Cratoxylum sp.

Ixonanthes icosandra

Ixonanthes icosandra

3०7'43.33', 101'39'10.97"

3०7'42.81', 101'39'10.29"

Ixonanthes icosandra

37'41.61', 101³9'11.71"

Ixonanthes icosandra

$3^{\circ} 7^{\prime} 40.47^{\prime \prime} \quad 101^{\circ} 39^{\prime} 1.04^{\prime \prime}$

$3^{\circ} 7^{\prime} 40.44^{\prime \prime} \quad 101^{\circ} 39^{\prime} 10.67^{\prime \prime}$

3०7'41.31', 101³9'14.46”

$3^{\circ} 7^{\prime} 40.43^{\prime \prime} \quad 101^{\circ} 39^{\prime} 13.52^{\prime \prime}$

3०7'42.88', 101'39'9.80"

37'41.55', 101'39'9.31"

3०7'41.83', 101'39'14.17'

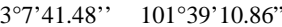

37'41.48', 101³9'11.41"

3०7'41.22', 101'39'10.70"

3०7'40.99', 101'39'11.19'

Cannarium sp. $\quad 37^{\circ} 40.70^{\prime \prime} 101^{\circ} 39^{\prime} 9.41^{\prime \prime}$

$\begin{array}{llll}\text { Cannarium sp. } & 3^{\circ} 7^{\prime} 40.76^{\prime}, & 101^{\circ} 39^{\prime} 12.00 ” & \text { Confirmed } \\ \text { Cannarium sp. } & 3^{\circ} 7^{\prime} 40.56^{\prime}, & 101^{\circ} 39^{\prime} 13.03^{\prime \prime} & \text { Confirmed }\end{array}$

Confirmed

Confirmed

Confirmed

Confirmed

Confirmed

Confirmed

Confirmed

Confirmed

Confirmed

Confirmed

Confirmed

Confirmed

Confirmed

Confirmed

Confirmed

Confirmed

Confirmed

Confirmed

Confirmed

Confirmed

Confirmed

Confirmed

Confirmed

Confirmed

Confirmed

Confirmed

Confirmed

Misclassified as

Ixonanthes sp.

Confirmed

Confirmed

Confirmed

Misclassified as

Ixonanthes sp.

Confirmed

Confirmed

Confirmed

Confirmed

Confirmed

Confirmed

Confirmed

Confirmed

Confirmed

Confirmed

Confirmed

Confirmed

Cannarium sp.

Confirmed 
Am. J. Applied Sci., 7 (4): 493-499, 2010

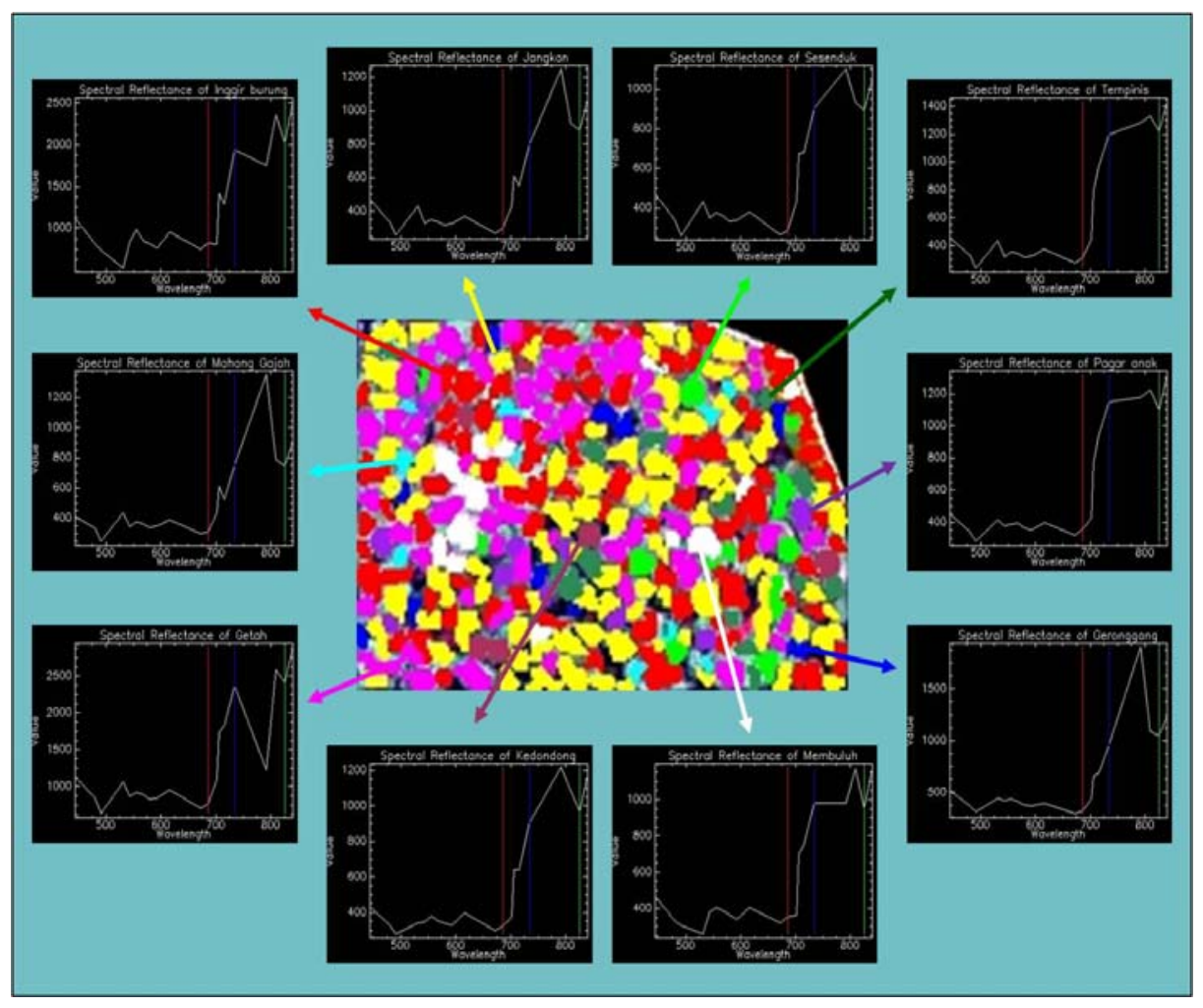

Fig. 4: Some selected image spectral signatures of the individual tree crowns developed from the UPM-TropAIR's AISA sensor data
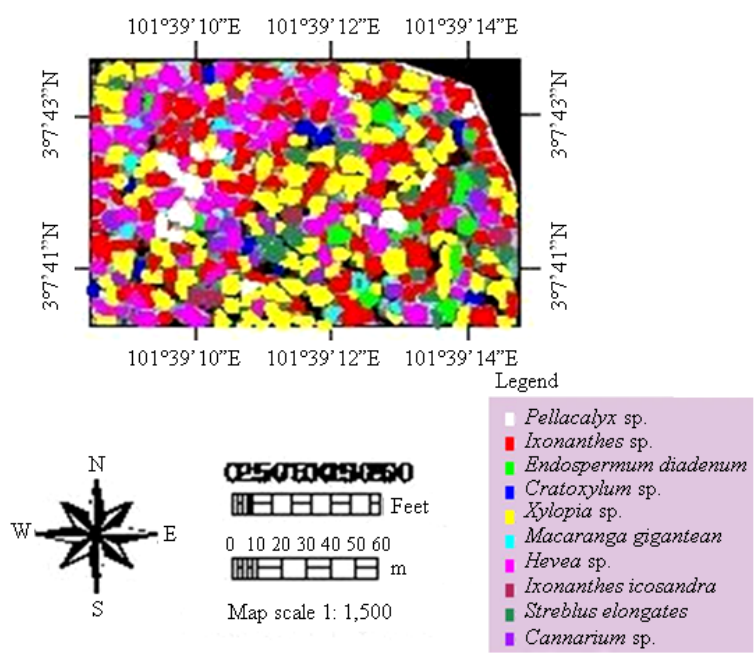

Fig. 5: The output thematic AeroMAP ${ }^{\mathrm{TM}}$ of forest in Taman Rimba Ilmu, University Malaya using UPM-TropAIR's AISA sensor

Table 1 shows the complete listing of the tree inventory amounting to a total of 297 trees as identified, quantified and mapped by the airborne sensor and later ground checked and verified by the field survey team using the GPS. Some selected image spectral reflectance of 10 randomly sampled tree crowns in the study area was shown in Fig. 4. Out of the total 297 trees mapped from the airborne sensor, 293 trees confirmed available on the ground and only 4 trees (Ixonanthes icosandra) was misclassified as Ixonanthes sp. and vice versa due to the very close proximity of its genus leading to a little confusion in the very similar spectral signature characteristics between the two genus. The mapping accuracy of this study was 98.65\% with the final thematic TropAIRMAP ${ }^{\mathrm{TM}}$ output product shown in Fig. 5.

\section{DISCUSSION}

Similar results of optimal band combinations for species identification were obtained from studies of (Jusoff and Ibrahim, 2009; Jusoff, 2009a) when they conducted studies on similar sensor applications to tropical forest species identification. Sobel edge detection filter showed the best effect on the image in detecting edges of the individual crowns or canopy of trees in Taman Rimba Ilmu's forest compared to 
other convolution and morphology filters. It is to be noted that there is no single standard method of enhancement can be said to be 'best' for all kind of images, the need of different user may differ (Mather, 1999). The individual species spectral reflectance's matching from the field versus image differed slightly due to the small variations in the species maturity during the collection of field data and GPS reading inaccuracies. This small variation in spectral reflectance has also been reported by Kamaruzaman et al. (2009)

\section{CONCLUSION}

UPM-TropAIR's AISA airborne sensor is able to precisely identify, quantify and map 297 individual tree crowns at the species level in Taman Rimba Ilmu, University Malaya with a mapping accuracy of $98.65 \%$. The tree crowns detected comprises of 83 Xylopia sp., 79 Ixonanthes sp., 56 Hevea sp., 15 Streblus elongates, 14 Pellacalyx sp., 12 Endospermum diadenum, 11 Macaranga gigantean, 10 Cratoxylum sp., 10 Cannarium sp. and 7 Ixonanthes icosandra.

\section{REFERENCES}

Darvishsefat, A.A., T.W. Kellenberger and K.I. Itten, 2002. application of hyperspectral data for forest stand mapping. Proceedings of the Symposium on Geospatial Theory, Processing and applications, (SGTPA'02)ISPRS, Ottawa, pp: 1-5.

http://www.isprs.org/commission4/proceedings02/ pdfpapers/357.pdf. Accessed on 3-10-2007

Jusoff, K. and H.M.H. M.Yusoff, 2008. Airborne hyper spectral imagery for agricultural businesses in Malaysia. J. Int. Bus. Res, 1: 54-62.

Jusoff, H.K. and N. Ya acob, 2008. Mapping of power transmission lines on Malaysian highways using UPM-APSB's AISA airborne hyper spectral imaging system. J. Comput. Inform. Sci., 1: 88-94.

Jusoff, K., 2006. Individual mangrove species identification and mapping in port Klang using airborne hyperspectral imaging. J. Sustain. Sci. Manage., 1: 27-36.

Jusoff, K., 2007. Advanced processing of UPMAPSB's AIS aairborne hyperspectral images for individual timber species identification and mapping. Int. J. Syst. Applied Eng. Dev., 2: 21-26.

Jusoff, K., 2008a. Development of geographic information system database for town planning of Sri Serdang, Malaysia using UPM-APSB's AISA airborne hyperspectral imaging data. The national mapping and spatial data committee (JPDSN), Malaysian national survey and mapping department. Bulletin GIS.
Jusoff, K., 2008b. Search And Rescue (SAR) operations for the missing bell 206 long ranger helicopter in Sarawak, Malaysia using near realtime airborne hyperspectral imaging systems. Disaster Preven. Manage. Int. J., 17: 94-103.

Jusoff, K., 2008c. Geospatial information technology for conservation of coastal forest and mangroves environment in Malaysia. Comput. Inform. Sci., 1: 129-134.

Jusoff, K. and K. Ibrahim, 2009. Hyper spectral remote sensing for tropical rainforest. Am. J. Applied Sci., 6: 2001-2005. DOI:10.3844/ajassp.2009.2001.2005

Jusoff, K. and P. Mubeena, 2009. Mapping of individual oil palm trees using airborne hyper spectral sensing: An Overview. Applied Phys. Res., 1: 15-30.

Jusoff, K., 2009a. Airborne hyper spectral sensor for individual species counting and mapping of Karas (Aquilaria malaccensis) in Bukit nanas F.R, Malaysia. World Applied Sci. J., 7: 1246-1251.

Jusoff, H.K., 2009b. Mapping of Sabah islands using airborne hyper spectrometer. J. Geography Geol., 1: 2-6.

Jusoff, K., 2009c. Precision forestry using airborne hyper spectral imaging sensor. J. Agric. Sci., 1: 142-147.

Jusoff, K., 2009d. Land use and cover mapping with airborne hyper spectral imager in situ, Malaysia. J. Agric. Sci., 1: 120-131.

Jusoff, K., 2010. Pixel-based airborne hyper spectral sensing technique for search and rescue of the missing RMAF NURI helicopter in GentingSempah, Malaysia. J. Disaster Prevention Manage., 19: 87-101.

Kamaruzaman, J., H.I. Mohd and H. Nurul, 2009. Spectral separability of tropical forest tree species using airborne hyper spectral imager. J. Environ. Sci. Eng., 3: 37-41.

Mather, P.M., 1999. Computer Processing of RemotelySensed Images: An Introduction. 2nd Edn., Wiley, West Sussex, ISBN: 0471985503, pp: 292.

Mohd Hasmadi, I., J. Kamaruzaman and M.A Nurul Hidayah, 2010. Analysis of crown spectral characteristic and tree species mapping of tropical forest using hyperspectral imaging. J. Trop. For. Sci., 22: 67-73.

Wilson, A.D., 2000. New methods, algorithms and software for rapid mapping of trees positions in coordinate forest plots. Department of Agriculture, Southern Research Station, Forest Service of United States, Washington. 